\title{
Atividades de autocuidado em pacientes com Diabetes Mellitus tipo 2: estudo transversal
}

\author{
Self-care activities in patients with type 2 Diabetes Mellitus: a cross-sectional study \\ Actividades de autocuidado en pacientes con diabetes mellitus tipo 2: estudio transversal
}

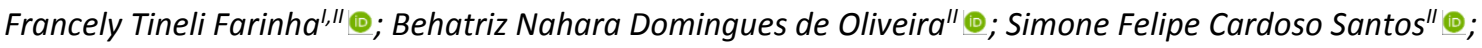

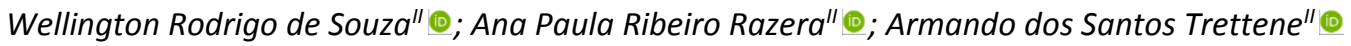 \\ 'Hospital de Reabilitação de Anomalias Craniofaciais, Universidade de São Paulo, Bauru, SP, Brasil; \\ "Universidade Paulista, Bauru, SP, Brasil
}

\begin{abstract}
RESUMO
Objetivo: avaliar as atividades de autocuidado em pacientes com Diabetes Mellitus tipo 2. Método: estudo transversal que incluiu 40 pacientes. Aplicou-se 2 instrumentos para coleta de dados: questionário sociodemográfico e questionário de atividades de autocuidado com o diabetes, aplicados entre janeiro e março de 2020. Resultados: a menor pontuação foi para o item "Realizar exercício físico específico (nadar, caminhar, etc)", com média 0,6 (DP=1,54) dias por semana, e a maior para o item "Tomar medicamentos conforme recomendados (insulina ou comprimidos)" com média 5,1 (DP=2,74) dias por semana. Quanto ao tabagismo, 92,5\% referiram não ter fumado cigarro nos últimos sete dias. Conclusão: as atividades de autocuidado prevalentes relacionaram-se as intervenções farmacológicas, enquanto os cuidados não farmacológicos, incluindo a alimentação e realização de exercícios físicos, foram menos frequentes.
\end{abstract}

Descritores: Doença crônica; Diabetes Mellitus Tipo 2; Autocuidado; Enfermagem.

\begin{abstract}
Objective: to evaluate self-care activities in patients with type-2 Diabetes Mellitus. Method: this cross-sectional study included 40 patients. Two instruments - a sociodemographic questionnaire and a questionnaire on diabetes self-care activities - were applied between January and March 2020. Results: the item "Perform specific physical exercise (swimming, walking, etc.)" scored lowest, averaging 0.6 (SD = 1.54) days a week, and "Taking medications as recommended (insulin or pills)" scored highest, with mean 5.1 (SD = 2.74) days a week. 92.5\% reported not having smoked cigarettes in the prior seven days. Conclusion: selfcare activities relating to pharmacological interventions predominated, while non-pharmacological care, including food and physical exercise, was less frequent.

Descriptors: Chronic disease; Diabetes Mellitus, Type 2; Self Care; Nursing.

\section{RESUMEN}

Objetivo: evaluar las actividades de autocuidado en pacientes con Diabetes Mellitus tipo 2. Método: este estudio transversal incluyó a 40 pacientes. Se aplicaron dos instrumentos, un cuestionario sociodemográfico y un cuestionario sobre actividades de autocuidado de la diabetes, entre enero y marzo de 2020. Resultados: el ítem "Realizar ejercicio físico específico (nadar, caminar, etc.)" obtuvo la puntuación más baja, con una media de 0,6 (DE = 1,54) días a la semana, y "Tomar los medicamentos según lo recomendado (insulina o píldoras)" obtuvo la puntuación más alta, con una media de 5,1 (DE = 2,74) días a la semana. El 92,5\% informó no haber fumado cigarrillos en los siete días anteriores. Conclusión: predominaron las actividades de autocuidado relacionadas con las intervenciones farmacológicas, mientras que la atención no farmacológica, incluida la alimentación y el ejercicio físico, fue menos frecuente.

Descriptores: Enfermedad Crónica; Diabetes Mellitus Tipo 2; Autocuidado; Enfermería.
\end{abstract}

\section{INTRODUÇÃO}

As doenças crônicas caracterizam-se por um longo curso clínico e, na maioria das vezes, por sua irreversibilidade. Sendo assim, constituem um problema de saúde pública, cuja persistência ao longo tempo requer gerenciamento de forma permanente e integral ${ }^{1}$.

Evidencia-se na atualidade, o aumento da morbimortalidade, do sedentarismo e do sobrepeso, redirecionando o perfil das patologias e corroborando com a persistência de doenças crônicas como o Diabetes Mellitus que se destaca pela elevada prevalência mundial e por altos índices de morbimortalidade. Em 2019, 463 milhões de pessoas encontravam-se com diabetes e este número está projetado para chegar a 578 milhões em 2030 e 700 milhões em 2045. Nas Américas Central e do Sul, estima-se 40 milhões de indivíduos com diabetes em $2030^{1,2}$.

O Diabetes Melittus tipo 2 caracteriza-se como uma doença crônica, cuja fisiopatogenia inclui fatores de controle modificáveis e não modificáveis. Dentre os modificáveis, destacam-se as atividades de autocuidado, incluindo mudanças no estilo de vida ${ }^{3}$. 
Em resumo, a pouca realização de atividades referente ao tratamento medicamentoso e não medicamentoso, agravam o quadro e favorecem o surgimento de complicações ${ }^{4}$.

Nesse sentido, destaca-se a importância das atividades de autocuidado que incluem mudanças de hábitos de vida e controle metabólico, como reeducação alimentar, introdução de atividade física, cuidados com o corpo e a mente, redução de danos com uso de álcool ou tabaco e uso adequado da terapia medicamentosa com hipoglicemiantes orais associados ou não a utilização da insulina ${ }^{5,6}$.

Sendo assim, estudos que permitam identificar as atividades de autocuidado de pacientes com esta doença são indispensáveis, principalmente por possibilitarem o planejamento e implementação de ações que favoreçam esse processo, com foco na promoção da saúde e na prevenção de complicações, além de promover a qualidade de vida e reduzir custos ao sistema de saúde.

Diante do exposto, questiona-se: quais as principais dificuldades referentes ao autocuidado são enfrentadas pelos pacientes com Diabetes Mellitus tipo 2? Nesse sentido, o presente estudo busca contribuir com a literatura e apresentar uma análise da caracterização desses usuários, descrevendo o perfil sociodemográfico clínico e terapêutico e as atividades de autocuidado de usuários com diabetes mellitus em um serviço de atenção básica à saúde.

Espera-se com este estudo, identificar os principais fatores, dúvidas e dificuldades enfrentadas pelos indivíduos acometidos por Diabetes Mellitus realizando assim um diagnóstico situacional, com a intenção de auxiliar e promover o autocuidado e consequentemente a melhora na qualidade de vida. Sendo assim, o objetivo deste estudo é avaliar as atividades de autocuidado em pacientes com Diabetes Mellitus tipo 2.

\section{MÉTOdo}

Trata-se de um estudo descritivo, transversal, de delineamento quantitativo, realizado entre janeiro e março de 2020.

A população foi composta por pacientes com diabetes tipo 2 acompanhados em uma Unidade de Estratégia da Saúde da Família de um município do interior de São Paulo, Brasil. Considerou-se para a construção da amostra os pacientes atendidos na unidade entre janeiro e março de 2020, ou seja, foram convidados a participar 58 pacientes. Destes, 40 aceitaram participar, constituindo a amostra deste estudo.

Os critérios de inclusão foram: apresentar idade igual ou superior a 18 anos, possuir Diabetes Mellitus tipo 2 e apresentar capacidade de comunicação verbal, ou seja, conseguirem expressar respostas coerentes com a pergunta realizada. Foram excluídos os usuários com déficit neurológico e cognitivo. Inicialmente os participantes foram convidados a participar, esclarecidos os objetivos da pesquisa e apresentados os instrumentos de coleta de dados.

A coleta de dados ocorreu em local reservado por meio de dois instrumentos: questionário sociodemográfico e o questionário de atividades de autocuidado com o diabetes (QAD) ${ }^{7}$.

Utilizou-se o questionário sociodemográfico para caracterizar os participantes segundo as variáveis: idade, sexo, escolaridade, número de filhos e estado civil ou afetivo. Para a avaliação do autocuidado utilizou-se o questionário de atividades de autocuidado com o diabetes (QAD) ${ }^{7}$.

Trata-se de um instrumento autoaplicável, que foi traduzido, adaptado e validado à cultura brasileira. O QAD avalia as atividades de autocuidado a partir de 07 dimensões e 17 itens, sendo: alimentação geral (dois itens), alimentação específica (três itens), atividade física (dois itens), monitorização da glicemia (dois itens), cuidado com os pés (três itens), uso da medicação (dois itens) e tabagismo (três itens) ${ }^{7}$.

Cada item é pontuado em uma escala do tipo Likert, cuja pontuação varia de zero a 7 pontos, onde o zero corresponde à situação menos desejável e o 7 a mais desejável, exceto na dimensão "alimentação específica" em que os valores são invertidos. O tabagismo é avaliado isoladamente, de acordo com a média de cigarros consumidos ao dia. $\mathrm{O}$ resultado avalia a frequência com que o paciente realizou as atividades, sendo assim, zero representa que o participante não realizou nenhuma atividade nos últimos sete dias. Em contrapartida, a pontuação sete significa que o paciente realizou as atividades diariamente nos últimos sete dias $^{7}$.

Os dados foram tabulados pelo programa Excel versão 2010 e submetidos a análise estatística descritiva na planilha Excel 2010.

A pesquisa foi aprovada pelo Comitê de Ética em Pesquisa da instituição por meio do ofício 3.767.067 e CAAE 25673419.6.0000.5512. Todos os participantes assinaram o Tremo de Consentimento Livre e Esclarecido, em consonância a Resolução 466/12 do Conselho Nacional de Saúde sobre pesquisas envolvendo seres humanos. 


\section{RESULTADOS}

Quanto à caracterização da amostra, a Tabela 1 apresenta a distribuição das variáveis pesquisadas.

\begin{tabular}{|c|c|c|c|}
\hline Variáveis & & $\mathbf{n}$ & $\%$ \\
\hline \multirow[t]{2}{*}{ Sexo } & Masculino & 11 & 27,5 \\
\hline & Feminino & 29 & 72,5 \\
\hline \multirow[t]{5}{*}{ Faixa etária } & $20-30$ anos & 1 & 2,5 \\
\hline & $31-40$ anos & 1 & 2,5 \\
\hline & $41-50$ anos & 6 & 15,0 \\
\hline & $51-60$ anos & 7 & 17,5 \\
\hline & $>60$ anos & 25 & 62,5 \\
\hline \multirow[t]{5}{*}{ Escolaridade } & Analfabeto & 8 & 20,0 \\
\hline & $\begin{array}{l}\text { Ensino Fundamental } \\
\text { Incompleto }\end{array}$ & 14 & 35,0 \\
\hline & Ensino Fundamental Completo & 4 & 10,0 \\
\hline & Ensino Médio Incompleto & 1 & 2,5 \\
\hline & Ensino Médio Completo & 11 & 27,5 \\
\hline \multirow[t]{4}{*}{ Tempo de Tratamento } & 1 anos -5 anos & 12 & 30,0 \\
\hline & 6 anos -10 anos & 11 & 27,5 \\
\hline & 11 anos -15 anos & 3 & 7,5 \\
\hline & $>15$ anos & 14 & 35,0 \\
\hline tal & & 40 & 100 \\
\hline
\end{tabular}

Prevaleceram os participantes do sexo feminino (72,5\%; $n=29)$, com idade maior que 60 anos $(62,5 \%$; $n=25)$, com ensino fundamental incompleto $(35,0 \% ; n=14)$ e em tratamento/acompanhamento por mais de 15 anos $(35,0 \% ; n=14)$.

Dos participantes, $85 \%(n=34)$ apresentavam outra doença além do Diabetes Mellitus, com predomínio da hipertensão arterial $(47,5 \% ; n=19)$.

Os dados investigados com o QAD são apresentados na Tabela 2.

TABELA 2: Avaliação dos itens do Questionário de Atividades de Autocuidado com o Diabetes na amostra estudada. Lençóis Paulista, São Paulo, Brasil. 2020.

\begin{tabular}{lcc}
\hline Dimensões do Questionário de Atividades de Autocuidado com Diabetes & $\begin{array}{l}\text { Média } \\
\text { (dias) }\end{array}$ & $\begin{array}{c}\text { Desvio } \\
\text { Padrão }\end{array}$ \\
\hline 1. Alimentação Geral & 1,9 & 2,39 \\
1.1 Seguir uma dieta Saudável & 2,43 \\
1.2 Seguir a orientação alimentar dada por um profissional & 1,4 \\
2. Alimentação Específica & 3,8 & 2,52 \\
2.1 Ingerir cinco ou mais porções de frutas e/ou vegetais & 1,96 \\
2.2 Ingerir carne vermelha e/ou derivados de leite integral & 2,9 \\
2.3 Ingerir doces & 3,2 & 2,29 \\
3. Atividade Física & 1,3 \\
3.1 Realizar atividade física por, pelo menos, 30 minutos & 2,43 \\
3.2 Realizar exercício físico específico (nadar, caminhar, etc) & 0,6 \\
4. Monitorização da Glicemia & 1,54 \\
4.1 Avaliar o açúcar no sangue & 2,8 \\
4.2 Avaliar o açúcar no sangue o número de vezes recomendado & 2,93 \\
5. Cuidados com os Pés & 1,6 \\
5.1 Examinar os pés & 2,45 \\
5.2 Examinar dentro dos sapatos antes de calçá-los & 3,1 \\
5.3 Secar os espaços entre os dedos dos pés, depois de lavá-los & 3,04 \\
6. Medicação & 2,3 & 2,89 \\
6.1 Tomar medicamentos conforme recomendados (insulina ou comprimidos) & 5,1 & 2,74 \\
6.2 Tomar insulina conforme recomendado & 4,8 & 2,28 \\
6.3 Tomar os comprimidos conforme recomendados & 5,7 & 2,50 \\
\hline
\end{tabular}


Quanto as dimensões que compõem o QAD, a menor pontuação para o item "Realizar exercício físico específico (nadar, caminhar, etc)", com média de 0,6 (DP=1,54) dias por semana, enquanto a maior pontuação relacionou-se ao item "Tomar medicamentos conforme recomendados (insulina ou comprimidos)", com média 5,1 (DP=2,74) dias por semana.

Observou-se ainda, que outras variáveis foram pouco pontuadas, incluindo: "Realizar atividade física por, pelo menos, 30 minutos" (média 1,3; DP=2,43), "Seguir a orientação alimentar dada por um profissional" (média 1,4; $\mathrm{DP}=2,43$ ), "Avaliar o açúcar no sangue o número de vezes recomendado" (média 1,6; DP2,45) e "Seguir uma dieta Saudável" (média 1,9; DP=2,39).

Na Tabela 3 apresentam-se dados relativos ao tabagismo nos últimos sete dias.

TABELA 3: Hábitos tabagísticos da amostra estudada, conforme itens do Questionário de Atividades de Autocuidado com o Diabetes. Lençóis Paulista, São Paulo, Brasil. 2020.

\begin{tabular}{lll}
\hline Questionário de Atividades de Autocuidado com Diabetes - tabagismo & $\mathbf{n}$ & $\mathbf{\%}$ \\
\hline Fumou cigarro durante os últimos 7 dias? & 37 & 92,5 \\
Não & 3 & 7,5 \\
Sim & &
\end{tabular}

Entre os participantes, $92,5 \%(n=37)$ referiram não terem fumado cigarro nos sete dias anteriores a coleta de dados. Contudo, 7,5\% ( $n=3)$ responderam afirmativamente, com uso entre 2 a 15 cigarros ao dia (média 9,6). Entre esses, ao serem questionados quanto a última vez em que haviam fumado, responderam "hoje".

\section{DISCUSSÃO}

Evidenciou-se neste estudo, quanto a caracterização dos participantes, uma população idosa, com prevalência de mulheres, com ensino fundamental incompleto, tempo médio da doença maior que 15 anos, com doenças associadas ao Diabetes Mellitus tipo 2, com destaque à Hipertensão Arterial.

Diferentes estudos apontam que as mulheres, por procurarem mais os serviços de saúde, são diagnosticadas com mais frequência em relação aos homens ${ }^{8-10}$.

Observou-se a prevalência de idosos entre os participantes, em conformidade à literatura ${ }^{10,11}$. A prevalência em idosos explica-se pelo fato de que a Diabetes Mellitus ser uma doença crônica e, consequentemente, afeta mais este tipo de população, em especial naqueles com mais de 65 anos de idade ${ }^{9}$.

O baixo nível de escolaridade encontrado neste estudo pode resultar em uma condição que favorece a realização inadequada do plano terapêutico por limitar o acesso a informações e a compreensão dos complexos mecanismos da doença e do tratamento, podendo aumentar os riscos à saúde ${ }^{12,13}$.

Outro aspecto em destaque relacionou-se ao tempo em que os participantes apresentavam o Diabetes Mellitus. Nesse sentido, um estudo demonstrou que o período da doença é uma variável relevante, visto que, quanto maior o tempo de diagnóstico, maior o risco de desenvolver complicações advindas de um controle metabólico insatisfatório ${ }^{14}$.

Como comorbidade associada, prevaleceu a hipertensão arterial sistêmica. Estudo realizado em 23 clínicas hospitalares na Inglaterra, Escócia e Irlanda do Norte que incluiu 4801 pessoas com Diabetes tipo 2, demonstrou associação significativa entre a incidência de complicações macro e microvasculares e elevação da pressão arterial em pacientes com diabetes ${ }^{15}$.

Esta morbidade constitui risco de doença cardiovascular, uma vez que a cardiopatia ocupa o segundo lugar dentre as complicações macro vasculares e está fortemente associada à mortalidade e morbidade de pessoas diabéticas ${ }^{16}$.

Referente as atividades de autocuidado, as práticas farmacológicas encontravam-se em destaque. Em contrapartida, as atividades físicas tiveram pontuações pouco expressivas. Este resultado aponta que a maior preocupação dos pacientes se relaciona ao tratamento medicamentoso. Demonstra ainda, que para os profissionais da saúde, gerenciar o esquema terapêutico e cumprir com o objetivo principal que é a obtenção dos níveis glicêmicos adequados e redução de complicações, seja de maior relevância ${ }^{16,17}$.

Destaca-se que pacientes com Diabetes Melittus tipo 2 apresentam risco aumentado de desenvolver complicações e comorbidades micro e macro vasculares, que só podem ser reduzidas se os pacientes participarem ativamente do manejo da doença e executarem as atividades de autocuidado de maneira satisfatória ${ }^{18}$.

Quanto as atitudes comportamentais de autocuidado nos participantes deste estudo, os baixos índices relacionaramse a alimentação saudável, avaliação da glicemia e cuidados com os pés, mesmo conhecendo a importância destas ações. 
Apesar do conhecimento acerca dos benefícios da realização destes comportamentos (criação de escolhas saudáveis, redução da gordura corporal, prevenção/controle da obesidade, níveis baixos de insulina circulante e prevenção ao pé diabético) os pacientes ainda apresentam grande dificuldade em adicionar novos hábitos em sua rotina ${ }^{19}$.

Considerando-se que o Diabetes Mellitus tipo 2 apresenta-se numa fase da vida na qual o indivíduo já possui hábitos bastante consolidados, torna-se relevante promover discussões e implementar estratégias que favoreçam assertivamente o controle da doença ${ }^{20}$.

Um fator preocupante relacionou-se a baixa realização da monitorização da glicemia capilar. Sabe-se que a hiperglicemia crônica é uma das principais causas do desenvolvimento de complicações sistêmicas ${ }^{21}$.

Resultado similar foi observado em outro estudo, que ao avaliar 25 pessoas com Diabetes Mellitus tipo 2 identificou que poucos realizavam o teste de glicemia capilar como forma de monitorar a glicemia. Contudo, sabe-se que para ocorrer a prevenção da hiperglicemia, hábitos saudáveis devem ser incorporados rotineiramente, tais como: alimentação, exercícios físicos e uso da medicação corretamente 22 .

Ressalta-se ainda, sobre a necessidade do estabelecimento de uma rotina de avaliação diária dos pés em indivíduos com diabetes, considerando que essa medida minimiza diversos problemas, como: infecção, ulceração, destruição de tecidos moles, associados a alterações neurossensoriais e doença arterial periférica ${ }^{23}$. Hábitos inadequados com os pés podem desencadear a formação de ulcerações que, e em casos mais graves, resultam em amputação do membro.

Nesse sentido, uma investigação que incluiu 171 pacientes com diabetes avaliou o conhecimento acerca de medidas preventivas e de autocuidado com os pés, cujos participantes eram exclusivamente idosos de baixa escolaridade, identificou que os participantes apresentavam baixo nível de conhecimento acerca das medidas preventivas e desconheciam hábitos importantes de autocuidado, tais como: uso de calçado adequado, avaliação dos pés rotineiramente e não hidratação entre os dedos dos pés ${ }^{24}$.

Em contrapartida, um aspecto positivo identificado neste estudo relacionou-se ao fato da grande maioria (92,5\%) dos participantes declararam-se não fumantes. De fato, essa parece ser uma tendência, pois um estudo realizado em Santa Catarina identificou a diminuição e baixa prevalência de fumantes entre pacientes com diabetes ${ }^{25}$. Sabe-se que o tabagismo aumenta a gordura visceral reduzindo a sensibilidade à insulina e elevando os níveis glicêmicos ${ }^{26}$.

Em resumo, observou-se um déficit importante referente as atividades de autocuidado nos pacientes com diabetes participantes deste estudo, apontando ser essa uma área carente de intervenções por parte dos profissionais de saúde, sabe-se que pacientes idosos e com diabetes apresentam baixos níveis de autocuidado e dificuldades no manejo de seus sintomas. Além disso, têm dificuldade em aderir a comportamentos de autocuidado continuadamente ${ }^{27}$.

Nesse sentido, o ideal é que o conteúdo das informações seja transmitido de forma simplificada, porém, que tenha impacto na vida da população, motivando-os a aprender sobre a doença e a assumir ativamente seu papel.

Assim, espera-se dos profissionais de saúde, especialmente os da enfermagem, esforços e envolvimento para disseminar e monitorar intervenções voltadas ao tratamento e autocuidado, considerando sempre os aspectos culturais e individuais de seus pacientes.

\section{Limitações do estudo}

A limitação deste estudo refere-se ao desenho transversal que impossibilita relações de causa e efeito. Além disso, deve-se considerar que o tamanho amostral limita a generalização dos resultados. Assim, são necessárias outras investigações nessa temática com amostras mais robustas e com delineamento prospectivo, que permitam consolidar os achados deste estudo.

\section{CONCLUSÃO}

Constatou-se que as atividades de autocuidado prevalentes se relacionaram as intervenções farmacológicas, enquanto os cuidados não farmacológicos, como alimentação e realização de exercícios físicos, foram menos frequentes. Frente aos resultados, o planejamento e a implementação de intervenções que visem promover ou estimular os cuidados não farmacológicos tornam-se imprescindíveis.

\section{REFERÊNCIAS}

1. Campolina AG, Adami F, Santos JLF, Lebrão ML. The health transition and changes in healthy life expectancy in the elderly population: possible impacts of chronic disease prevention. Cad. Saúde Pública [Internet]. 2013 [cited 2019 Nov 10]; 29(6):1217-29. DOI: https://doi.org/10.1590/S0102-311X2013000600018.

2. International Diabetes Federation. IDF Diabetes Atlas - Seventh Edition, 2019. [Internet]; Brussels, Belgium: International Diabetes Federation; 2019 [cited 2019 Dec 10]; 168p. Available from: http://www.diabetesatlas.org. 
3. Iquize RCC, Theodoro FCET, Carvalho KA, Oliveira MA, Barros JF, Silva AR. Educational practices in diabetic patient and perspective of health professional: a systematic review. J. Bras. Nefrol. [Internet]. 2017 [cited 2019 Nov 12]; 39(2):196-204. DOI: https://doi.org/10.5935/0101-2800.20170034.

4. Corrêa K, Gouvêa GR, Silva MAV, Possobon RF, Barbosa LFLN, Pereira AC, et al. Quality of life and characteristics of diabetic patients. Ciênc. saúde coletiva [Internet]. 2017 [cited 2019 Nov 12]; 22(3):921-30. DOI: https://doi.org/10.1590/141381232017223.24452015.

5. Silva SA, Alves SHS. Knowledge about type 2 diabetes and its relationship with the adherence to treatment. Est. Inter. Psicol. [Internet]. 2018 [cited 2019 Nov 10]; 9(2):39-57. Available from: http://pepsic.bvsalud.org/scielo.php?script=sci_arttext\&pid=\$2236-64072018000200004\&lng=es\&tlng=pt.

6. Rossi VEC, Silva AL, Fonseca GSS. Adherence to drug treatment among people with type 2 diabetes mellitus. Rev enferm CentOeste Min [Internet]. 2015 [cited 2019 Nov 10]; 5(3):1820-30. Available from: http://seer.ufsj.edu.br/index.php/recom/article/view/890/934.

7. Michels MJ, Coral MHC, Sakae TM, Damas TB, Furlanetto LM. Questionnaire of diabetes self-care activities: translation, crosscultural adaptation and evaluation of psychometric properties. Arq. Bras. Endocrinol. Metab. [Internet]. 2010 [cited 2019 Nov 16]; 54(7):644-51. DOI: https://doi.org/10.1590/S0004-27302010000700009.

8. Sarno F, Bittencourt CAG, Oliveira SA. Profile of patients with hypertension and/or diabetes mellitus from Primary Healthcare units. Einstein [Internet]. 2020 [cited 2020 jun 26]; 18:1-6. DOI: https://doi.org/10.31744/einstein_journal/2020ao4483.

9. Flor LS, Campos MR. The prevalence of diabetes mellitus and its associated factors in the Brazilian adult population: evidence from a population-based survey. Rev. Bras epidemiol. [Internet]. 2017 [cited 2020 Jun 26]; 20(1):16-29. Available from: https://www.scielosp.org/pdf/rbepid/2017.v20n1/16-29.

10. Santos GM, Sousa PVL, Barros NVA. Epidemiological profile of diabetic seniors registered in the Hiperdia Program in the state of Piauí, Brasil. Rev. Aten. Saúde [Internet]. 2018 [cited 2020 Jun 26]; 16(56):48-53. Available from: https://seer.uscs.edu.br/index.php/revista_ciencias_saude/article/view/5090/pdf.

11. Moreschi C, Rempel C, Siqueira DF, Backes DS, Pissaia LF, Grave MTQ. Family health strategies: profile/quality of life of people with diabetes. Rev. Bras. Enferm. [Internet]. 2018 [cited 2020 Jun 26]; 71(6):3073-80. DOI: http://dx.doi.org/10.1590/00347167-2018-0037.

12. Salin AB, Bandeira MSN, Freitas PRNO, Serpa I. Diabetes Mellitus tipo 2: population profile and factors associated with therapeutic adherence in Basic Health Units in Porto Velho-RO. REAS [Internet]. 2019 [cited 2020 Jun 26]; 33:e1257. DOI: https://doi.org/10.25248/reas.e1257.2019.

13. Figueira ALG, Boas LCGV, Coelho ACM, Freitas MCF, Pace AE. Educational interventions for knowledge on the disease, treatment adherence and control of diabetes mellitus. Rev. Latino-Am. Enfermagem [Internet]. 2017 [cited 2020 Jun 26]; 25:e2863. DOI: https://doi.org/10.1590/1518-8345.1648.2863.

14. Rodrigues FFL, Santos MA, Teixeira CRS, Gonela JT, Zanetti ML. Relationship between knowledge, attitude, education and duration of disease in individuals with diabetes mellitus. Acta paul. enferm. [Internet]. 2012 [cited 2019 Nov 22]; 25(2):284-90. DOI: https://doi.org/10.1590/S0103-21002012000200020.

15. Adler Al, Stratton IM, Neil HAW, Yudkin JS, Matthews DR, Cull Ca et al. Association of systolic blood pressure with macrovascular and microvascular complications of type 2 diabetes (UKPDS 36): prospective observational study. BMJ [Internet]. 2000 [cited 2019 Nov 22]; 321:412-9. DOI: https://doi.org/10.1136/bmj.321.7258.412.

16. Oliveira REM, Ueta J, Franco LJ. Adherence to medication treatment of type 2 diabetes mellitus: gender diferences. Rev. APS [Internet]. 2018 [cited 2020 Jan 22]; 21(3):335-44. DOI: https://doi.org/10.34019/1809-8363.2018.v21.16395.

17. Rossi VEC, Silva AL, Fonseca GSS. Adherence to drug treatment among people with type 2 diabetes mellitus. Rev. enferm. CentOeste Min. [Internet]. 2015 [cited 2019 nov 22]; 5(3):1820-30. Available from: http://www.seer.ufsj.edu.br/index.php/recom/article/view/890.

18. Moura NS, Lopes BB, Teixeira JJD, Oriá MOB, Vieira NFC, Guedes MVC. Literacy in health and self-care in people with type 2 diabetes mellitus. Rev. Bras. Enferm. [Internet]. 2019 [cited 2020 Jan 22]; 72(3):734-40. DOI: https://doi.org/10.1590/00347167-2018-0291.

19. Souza KOC, Mendonça SCB, Otero LM, Souza MFC, Ribeiro SO. Self-care of patients with Diabetes Mellitus type 2. Semina cienc. boil. saude [Internet]. 2019 [cited 2020 Jan 22]; 40(1):75-88. DOI: http://dx.doi.org/10.5433/1679-0367.2019v40n1p75.

20. Oliveira GYM, Almeida AMO, Girão ALA, Freitas CHA. Nursing interventions for promoting self-care of persons with type 2 diabetes: an integrative review. Rev. Eletr. Enf. [Internet]. 2016 [cited 2019 Nov 22]; 18:e1188. DOI: http://dx.doi.org/10.5216/ree.v18.38691.

21. Stehouwer CDA. Microvascular dysfunction and hyperglycemia: a vicious cycle with widespread consequences. Diabetes [Internet]. 2018 [cited 2019 Nov 22]; 67(9):1729-41. DOI: https://doi.org/10.2337/dbi17-0044.

22. Baptista MHB, Dourado FC, Gomides DS, Teixeira CRS, Freitas MCF, Pace AE. Education in Diabetes Mellitus for blood glucose self-monitoring: a quasi-experimental study. Rev. Bras. Enferm. [Internet]. 2019 [cited 2020 Jan 22]; 72(6):1679-86. DOI: http://dx.doi.org/10.1590/0034-7167-2018-0731.

23. Neto MO, Pereira MS, Pinto MAH, Agostinho LM, Júnior FER, Hissa MN. Evaluation of self-care for diabetic foot prevention and clinical examination of the feet in a diabetes mellitus reference center. J. Health Biol. Sci. [Internet]. 2017 [cited 2019 Nov 22]; 5(3):265-71. DOI: http://dx.doi.org/10.12662/2317-3076jhbs.v5i3.1092.p265-271.2017.

24. Sousa VM, Sousa IA, Moura KR, Lacerda LSA, Ramos MGS, Silva ARV. Knowledge about preventive measures for the development of diabetic foot. Rev. Rene [Internet]. 2020 [cited 2019 Jun 22]; 21:e42638. DOI: https://doi.org/10.15253/21756783.20202142638 
25. Hoepers NJ, Roldão GS, Fernandes PR, Dimer LM, Pavei SRP. Self-care of people with diabetes mellitus type II in family health strategy. Inova Saúde [Internet]. 2018 [cited 2019 Jun 22]; 8(2):116-37. DOI: http://dx.doi.org/10.18616/inova.v8i2.3458.

26. Campagna D, Alamo A, Pino AD, Russo C, Calogero AE, Purrello F et al. Smoking and diabetes: dangerous liaisons and confusing relationships. Diabetol. Metab. Syndr. [Internet]. 2019 [cited 2020 Jun 22]; 11:85. DOI: https://doi.org/10.1186/s13098-0190482-2.

27. Kim MY, Lee EJ. Factors affecting self-care behavior levels among elderly patients with type 2 diabetes: a quantile regression approach. Medicina (Kaunas) [Internet]. 2019 [cited 2020 Jun 22]; 55(7):340. DOI: https://doi.org/10.3390/medicina55070340. 